\title{
Análise de estudos qualitativos conduzidos por médicos publicados em periódicos científicos brasileiros entre 2004 e 2013
}

| ${ }^{1}$ Stella Regina Taquette, ${ }^{2}$ Maria Cecília Minayo |

Resumo: Analisamos artigos de pesquisas realizadas por médicos que utilizaram método qualitativo. A busca bibliográfica foi feita na base SciELO do Brasil no período de 2004 a 2013 com as palavras-chave: pesquisa qualitativa, entrevista, grupo focal, observação participante, análise de conteúdo, análise de discurso, representação social, hermenêutica-dialética. Abordamos os textos por meio de constructo teórico baseado nas diretrizes RATS para revisão de estudos qualitativos.

Classificamos o material nas categorias: consistente, pouco consistente e inconsistente. Selecionamos 135 artigos de 28 periódicos. Consideramos a maioria consistente $(64,4 \%)$. Os principais problemas encontrados foram: ausência de informações sobre percurso metodológico; análise parcial e descritiva, sem diálogo com a literatura; conclusões que não avançam além do senso comum; resultados descontextualizados e limitaçôes do estudo não consideradas. Concluímos que a maior parte dos artigos analisados tem validade científica e sugerimos a inclusão de normas para esse tipo de publicação nos periódicos da área de saúde.

> Palavras-chave: pesquisa qualitativa; conhecimento científico; epistemologia; avaliação por pares.

\author{
1 Faculdade de Ciências \\ Médicas, Universidade do \\ Estado do Rio de Janeiro. Rio \\ de Janeiro-RJ, Brasil (stella. \\ taquette@gmail.com). \\ 2 Escola Nacional de Saúde \\ Pública, Fundação Oswaldo \\ Cruz. Rio de Janeiro-RJ, Brasil \\ (cecilia@claves.fiocruz.br).
}

Recebido em: 21/07/2015 Aprovado em: 15/12/2015 


\section{Introdução}

A maioria dos estudos científicos que utiliza o método qualitativo na área de saúde não é desenvolvida por médicos, apesar destes, em teoria, serem atores privilegiados para a realização de investigações dessa natureza graças a sua proximidade com as pessoas (TURATO, 2005). Também são escassos os periódicos da área da Medicina que publicam pesquisas de cunho qualitativo. $\mathrm{O}$ método qualitativo de pesquisa é aqui entendido como aquele que se ocupa do nível subjetivo e relacional da realidade social e é tratado por meio da história, do universo, dos significados, dos motivos, das crenças, dos valores e das atitudes dos atores sociais (MINAYO, 2013). Esse método é quase desconhecido ou pouco valorizado pelos médicos, pois há uma lacuna na formação médica no que diz respeito à área das Ciências Humanas, além desses profissionais considerarem que a produção qualitativa se presta pouco à reprodutibilidade e à generalização dos resultados (TAQUETTE; MINAYO; RODRIGUES, 2015).

Morse (2006), editora da revista Qualitative Health Research, comenta que pesquisadores médicos consideram a investigação qualitativa muito subjetiva, tendenciosa e, por isso, sem validade científica. Canesqui (2011, p. 18), em suas reflexôes sobre a presença das Ciências Sociais e Humanas na Saúde Pública argumenta que "pode-se dizer que a Medicina continua ensurdecida à pessoa humana”, ao comentar a dificuldade que os profissionais dessa área têm de dar atenção aos aspectos subjetivos da realidade das pessoas e ao contexto social. Por sua vez, os textos científicos sobre pesquisa qualitativa costumam utilizar jargões das Ciências Sociais, pouco usuais aos médicos (MARSIGLIA, 2013). Os cientistas sociais sentem dificuldade de "traduzir" a linguagem de suas áreas, de forma a torná-la acessível aos profissionais das áreas da saúde, dificultando uma integração interdisciplinar e fazendo com que esses últimos não alcancem um olhar mais complexo sobre os fenômenos do adoecimento, da vida e da morte. No entanto, os novos currículos dos cursos de Medicina contemplam as disciplinas humanísticas e sociais, embora a ênfase e o interesse de professores e estudantes permaneçam voltados para matérias que compõem o modelo biomédico tecnicista, dificultando a construção de um olhar integral para o ser humano que sofre com doenças e agravos.

O número de publicações sobre pesquisas qualitativas em saúde tem aumentado, o que é uma boa notícia. Porém, isso vem ocorrendo num ritmo 
menor do que a multiplicação de artigos com abordagens quantitativas. Não raro, profissionais das Ciências Sociais engajados no setor saúde alertam sobre a escassez de publicações e também sobre a pouca consistência teórica observada na interpretação dos dados coletados (GOMES; SILVEIRA, 2012). Tais observações não se referem apenas à produção nacional, pois Minayo (2012a), num ensaio que se baseia em textos dos autores contemporâneos mais renomados das Ciências Sociais no mundo, encontrou que, no meio deles, também há uma controvérsia importante entre os que defendem os estudos empíricos per se como o modo essencial desse tipo de ciência e os que ressaltam seu papel teórico e crítico. No primeiro caso, vários desses autores argumentam sobre a necessidade de certo pragmatismo nas pesquisas para dar soluções aos problemas concretos da sociedade. Em carta ao editor da Revista de Saúde Pública, Oliveira et al. (2012) levantam discussão sobre maneiras de fazer ciência na pesquisa qualitativa. Os autores tecem críticas à banalização do uso dos termos sem o necessário aprofundamento conceitual e epistemológico, o que prejudica a compreensão do objeto e compromete a validade de seus resultados. A divulgação de estudos com consistência questionável é um estorvo para consolidação do método qualitativo como relevante produtor de conhecimento.

Neste artigo, perguntamo-nos se têm consistência metodológica os trabalhos derivados de pesquisas qualitativas em saúde escritos por médicos e publicados em periódicos científicos indexados. O objetivo desta revisão bibliográfica é identificar pontos fortes e fragilidades nesses artigos e, assim, verificar a validade do conhecimento produzido que é divulgado, tendo como base teórica da análise as diretrizes RATS de revisão de pesquisa qualitativa.

\section{Percurso metodológico}

Realizamos uma busca de artigos publicados em periódicos indexados no Scientific Electronic Library Online (SciELO) do Brasil entre os anos de 2004 e 2013, desenvolvidos por médicos e com método qualitativo. Selecionamos essa base de dados pelo fato de ser o mais completo acervo das revistas científicas brasileiras. As publicações que estão nessa base passam por avaliação periódica realizada por comitê de profissionais experientes e representantes das grandes áreas de conhecimento. Não menos importante, os artigos publicados nesse conjunto de periódicos são obrigatoriamente avaliados por pares. 
$\mathrm{Na}$ busca dos artigos, utilizamos as seguintes palavras-chave para identificar estudos qualitativos: entrevista (em profundidade, semiestruturada, aberta); pesquisa/método/estudo qualitativo(a); grupo focal; história de vida; história oral; observação participante; representaçōes sociais; narrativa(s); análise de conteúdo; análise de discurso; hermenêutica e dialética. Esse primeiro critério de seleção deu origem a 1616 artigos. Em seguida, os selecionamos quanto ao título e ao periódico em que foram publicados. Para ampliar a chance de encontrar autores médicos, foram eliminados os títulos que claramente demonstravam que o tema do estudo ou do periódico era de área distinta da saúde (linguística, economia, política, entre outras). Também foram descartados, pela mesma razão, os publicados em periódicos de áreas como enfermagem e odontologia, que tradicionalmente veiculam artigos de profissionais dessas categorias. Após esses dois filtros, lemos os resumos dos artigos. Quando o manuscrito dizia respeito à pesquisa realizada no campo da saúde, buscamos os currículos do primeiro e segundo autor na Plataforma Lattes do CNPq para identificação de médicos. Além dessa estratégia, selecionamos artigos de médicos conhecidos na área qualitativa, indicados por outros pesquisadores e também outros trabalhos de médicos já identificados. Excluímos da análise os artigos somente conceituais que não eram fruto de pesquisa documental ou de campo.

Analisamos os artigos selecionados tendo como base teórica as diretrizes RATS de revisão de pesquisa qualitativa (BIOMED, 2014). Estas diretrizes foram escolhidas por serem recomendadas aos revisores de manuscritos pela Revista de Saúde Pública, de São Paulo, um dos periódicos brasileiros mais bem qualificados pelos critérios da CAPES, tendo classificação A2 em 2014 em Saúde Coletiva, área predominante dos artigos pesquisados. As diretrizes são compostas por quatro conjuntos de itens aqui resumidos: (I) $\mathbf{R}$ - relevância justificada da questão em estudo; (II) A - adequação da metodologia qualitativa; (III) $\mathbf{T}$ transparência dos procedimentos; e (IV) $\mathbf{S}$ - solidez na abordagem compreensiva e interpretativa. A partir delas criamos uma tabela de pontuação com 15 itens descritos na tabela 1 , na tentativa de uniformizar e reduzir a subjetividade da avaliação, classificando-os em três categorias: A - consistentes, quando atendiam suficientemente de 12 a 15 itens; B - parcialmente analisados, apenas descritivos ou pouco consistentes, quando não havia transparência metodológica ou as interpretaçôes eram insuficientes, atendendo de 8 a 11 itens; e $\mathrm{C}$ - inconsistentes 
ou não analisados, quando atendiam sete ou menos itens. Sabemos, entretanto,

que estas diretrizes têm limitações quanto à análise da complexidade e profundidade teórica envolvida nas pesquisas de natureza qualitativa. Contudo, acreditamos que dão uma contribuição positiva na avaliação do manuscrito no que diz respeito aos itens constantes das diretrizes.

Lemos e relemos os artigos em dois momentos diferentes para análise e classificação, de acordo com as categorias contidas na tabela, com um intervalo de dois meses entre a primeira e a segunda avaliação. Acreditamos que a reanálise e a reclassificação dos artigos contribuíram para reduzir possíveis vieses e discordâncias entre os dois momentos de avaliação.

Tabela 1. Referencial para análise de consistência de artigos qualitativos

\begin{tabular}{|c|l|l|}
\hline $\mathbf{R}$ & Relevância justificada da questão do estudo & 3 pontos \\
\hline 1 & definição clara do objeto e do objetivo em estudo & \\
\hline 2 & quadro teórico referencial consistente e com pressupostos & \\
\hline 3 & objeto de estudo relevante & $\mathbf{3}$ pontos \\
\hline A & Adequação da metodologia qualitativa & \\
\hline 4 & método escolhido justificado & \\
\hline 5 & instrumentos utilizados adequados incluindo-se itens do roteiro & $\mathbf{4}$ pontos \\
\hline 6 & critérios de inclusão explícitos & \\
\hline T & Transparência dos procedimentos & \\
\hline 7 & cenário do estudo/estratégia de entrada no campo & \\
\hline 8 & descrição sobre como se deu a coleta de dados /amostragem & \\
\hline 9 & descrição de como se deu o registro da coleta de dados & \\
\hline 10 & aspectos éticos (incluindo o papel do pesquisador) & \\
\hline S & Solidez da abordagem interpretativa & \\
\hline 11 & análise apropriada, como foi decomposto o material analisado & \\
\hline 12 & contextualização histórico-espacial-social & \\
\hline 13 & $\begin{array}{l}\text { interpretações claras, apoiadas na evidência e com diálogo com a } \\
\text { literatura atualizada }\end{array}$ & \\
\hline 14 & limitações descritas & \\
\hline 15 & texto bem escrito, sem jargões & \\
\hline & TOTAL & \\
\hline & & \\
\hline & & \\
\hline
\end{tabular}




\section{Resultados}

Encontramos 135 artigos conduzidos por médicos, 82 como autores e 53 como coautores. Os textos com abordagem qualitativa na área da saúde são minoria, conforme comprovado em outros estudos (HOFF; WITT, 2000; MCKIBBON; GADD, 2004; WEINER et al., 2011; YAMAZAKI et al., 2009). Apesar da variedade de palavras-chave utilizadas, estamos certas de que não mapeamos todos os estudos qualitativos publicados por médicos, pois muitos não dispõem de descritores que os identifiquem como tal. Os reconhecidos na busca são de médicos em 12 áreas de atuação, sendo as duas mais frequentes Saúde Pública $(38,5 \%)$ e Psiquiatria $(34,8 \%)$ e as outras: medicina de adolescentes, medicina de família, gineco-obstetrícia, clínica médica, pediatria, homeopatia, saúde indígena, geriatria, dermatologia e hematologia.

Os artigos foram publicados em 28 periódicos de duas grandes áreas, Ciências da Saúde (a maioria - 25) e Ciências Humanas (3), sendo que sete continham $75 \%$ das publicações analisadas. São eles, por ordem decrescente segundo número de artigos: Ciência \& Saúde Coletiva, Cadernos de Saúde Pública, Revista de Saúde Pública, Physis, Interface, Revista Brasileira de Educação Médica e Saúde e Sociedade. Na tabela 2 pode ser visualizada a distribuição dos periódicos segundo o número de artigos qualitativos pesquisados.

Dos 28 periódicos em que os médicos escrevem, apenas cinco têm normas editoriais explícitas sobre publicação de estudos qualitativos. Alguns informam, por exemplo, que são aceitos "outros formatos de artigos originais quando pertinentes, de acordo com a natureza do trabalho". Oito não contêm informações relativas à aceitação de textos qualitativos, mas permitem artigos longos, o que facilita a publicação de estudos dessa natureza, que, em geral, ocupam mais espaço. Seis dispõem, em suas instruções para autores, normas que sugerem a rejeição de estudos qualitativos como: "deve-se explicar os procedimentos realizados, de tal modo que outros pesquisadores possam repetir o estudo; o procedimento estatístico utilizado deve ser descrito". Oito só aceitam artigos curtos, isto é, inibem os qualitativos. Um periódico apresenta normas ambíguas, pois ao mesmo tempo em que informa que os estudos devem apresentar resultados que possam ser replicados ou generalizados, admite tratamento estatístico ou categorização de dados. Periódicos internacionais, em levantamento desenvolvido por Hoff e Witt (2000), também não explicitam um número padrão de páginas dedicadas a 
esse tipo de estudo ou evidências de que haja a compreensão de que eles precisam de mais espaço por serem de natureza dissertativa.

Tabela 2. Distribuição dos periódicos segundo a grande área de conhecimento e o número de artigos qualitativos pesquisados

\begin{tabular}{|l|l|c|}
\hline PERIÓDICO & GRANDE ÁREA & No ARTIGOS \\
\hline 1. Ciência \& Saúde Coletiva & Ciências da Saúde & 24 \\
\hline 2. Cadernos de Saúde Pública & Ciências da Saúde & 20 \\
\hline 3. Revista de Saúde Pública & Ciências da Saúde & 16 \\
\hline 4. Physis & Ciências da Saúde & 13 \\
\hline 5. Interface & Ciências da Saúde & 10 \\
\hline 6. Rev. Brasileira de Educação Médica & Ciências da Saúde & 10 \\
\hline 7. Saúde e Sociedade & Ciências da Saúde & 8 \\
\hline 8. Revista Brasileira de Psiquiatria & Ciências da Saúde & 4 \\
\hline 9. Rev. Bras. Saúde Materno-Infantil & Ciências da Saúde & 4 \\
\hline 10. Rev. Brasileira de Saúde Ocupacional & Ciências da Saúde & 2 \\
\hline 11. São Paulo Medical Journal & Ciências da Saúde & 2 \\
\hline 12. Trends Psychiatric and Psychotherapy & Ciências da Saúde & 2 \\
\hline 13. Estudos de Psicologia & Ciências Humanas & 2 \\
\hline 14. Revista Brasileira de Enfermagem & Ciências da Saúde & 2 \\
\hline 15. Rev. Lat.-Am. Psicologia Fundamental & Ciências Humanas & 2 \\
\hline 16. Revista de Psiquiatria do R.G. do Sul & Ciências da Saúde & 2 \\
\hline 17. Acta Amazônia & Ciências da Saúde & 1 \\
\hline 18. Jornal Brasileiro de Nefrologia & Ciências da Saúde & 1 \\
\hline 19. Psicologia em Estudo & Ciências Humanas & 1 \\
\hline 20. Revista da Assoc. Médica Brasileira & Ciências da Saúde & 1 \\
\hline 21. Revista Brasileira de Hematologia & Ciências da Saúde & 1 \\
\hline 22. Estudos Feministas & Ciências da Saúde & 1 \\
\hline 23. Revista de Nutrição & Ciências da Saúde & 1 \\
\hline 24. Revista de Psiquiatria Clínica & Ciências da Saúde & 1 \\
\hline
\end{tabular}




\begin{tabular}{|l|c|c|}
\hline PERIÓDICO & GRANDE ÁREA & No ARTIGOS \\
\hline 25. Escola de Enfermagem Anna Nery & Ciências da Saúde & 1 \\
\hline 26. Texto e Contexto de Enfermagem & Ciências da Saúde & 1 \\
\hline 27. Saúde em Debate & Ciências da Saúde & 1 \\
\hline 28. Rev. Bras. Geriatria e Gerontologia & Ciências da Saúde & 1 \\
\hline TOTAL & & 135 \\
\hline
\end{tabular}

Abaixo apresentamos os dados dos artigos por categoria $\mathrm{A}, \mathrm{B}$ e $\mathrm{C}$ e, em seguida, as principais fragilidades encontradas em cada um dos quatro conjuntos de itens, segundo a tabela baseada nas diretrizes RATS utilizada na análise.

\section{Estudos consistentes}

Os artigos classificados na categoria A corresponderam a 64,4\% do total, ou seja, nossa avaliação conferiu qualidade à maioria dos estudos. Alguns, apesar de produzirem conhecimento relevante e original, saíram da categoria A por não atingirem pontuação segundo os critérios estabelecidos. Entre os artigos da categoria $A$, nenhum recebeu a pontuação total dos 15 itens da tabela. Os pontos menos contemplados foram "contextualização histórico-espacial-social” e "limitações do estudo". Vale destacar que poucos artigos apontam as limitações do estudo e muitos dos que contemplam esse item confundem "limitaçôes do estudo" com "limitações do método", como se pode observar em trechos retirados de alguns manuscritos analisados:

A presente pesquisa tem limitações de generalização dos estudos qualitativos.

Como em qualquer pesquisa qualitativa, as interpretações feitas no presente texto podem ser consideradas válidas internamente à amostra de sujeitos abordados.

Ora, essas duas frases dizem respeito a especificidades do método qualitativo e não às limitações reconhecidas pelo autor em seu texto.

\section{Estudos pouco consistentes ou parcialmente analisados}

Foram 32,6\% os artigos classificados na categoria B. Desses, a maioria $(54,5 \%)$ perde por consistência metodológica e 45,4\%, demonstram fragilidade quanto à análise e interpretação dos resultados; na sua maioria, apresentam falhas quanto à transparência dos procedimentos, não explicitando, por exemplo, o 
embasamento teórico das categorias apresentadas ou como foi decomposto o material analisado. Os itens menos pontuados, em ordem decrescente, foram "descrição de como se deu o registro da coleta de dados", "descrição de como foi feita a coleta de dados" e "aspectos éticos". Houve artigos em que os autores não falam sobre a amostragem, a duração das entrevistas, quem as realizou ou se foram gravadas. Quanto aos aspectos éticos, a maioria dos artigos que o relata apresenta aprovação do Comitê de Ética em Pesquisa da instituição de origem e a assinatura do Termo de Consentimento Livre e Esclarecido.

Os artigos considerados parcialmente analisados possuíam fragilidades principalmente nos itens "contextualização", "interpretação" e "limitações do estudo." Muitos não avançam além do senso comum, contentando-se em explicitar a opinião dos interlocutores: apresentam dados empíricos como se fossem a verdade sobre o grupo pesquisado. Inferimos, pelos pontos tratados acima, que os textos classificados como "pouco consistentes ou parcialmente analisados" seriam de melhor qualidade caso houvesse uma política editorial que enfatizasse a importância dos estudos qualitativos e apresentasse diretrizes específicas para sua avaliação.

\section{Estudos inconsistentes}

Classificamos na categoria C, como inconsistentes, apenas 3\% dos artigos. Acreditamos que, assim como bons artigos qualitativos eventualmente são rejeitados por pareceristas com pouca experiência no campo, artigos mal elaborados também são aceitos, pelo mesmo motivo. Entre os classificados nessa categoria encontram-se os que não justificam o objeto, não têm elaboração teórica, apresentam inadequação dos instrumentos de pesquisa. Em alguns, o autor chega a responsabilizar o interlocutor pela escassez das informaçóes coletadas.

Destacamos certas inadequações identificadas na leitura dos artigos por considerar que elas comprometem sua validade e cientificidade. Alguns apresentam falhas na formatação e padronização do estudo. Por exemplo, os autores descrevem os objetivos e os resultados dentro da metodologia. Outros apresentam erros conceituais, como informar que foi feita análise de discurso, quando na apresentação dos resultados verifica-se que o autor trabalhou com análise de conteúdo. Há também menção a tipos de abordagem qualitativa que não se adequam ao tipo de estudo, a exemplo de um cujo autor menciona o uso da técnica de história oral quando, na verdade, utilizou e era pertinente a entrevista aberta clássica. 
Justificativa da relevância da questão em estudo - Os artigos avaliados apresentaram neste primeiro conjunto de itens poucas fragilidades. $\mathrm{O}$ item "objeto em estudo relevante" não foi pontuado em apenas 10 artigos (7,5\%), porque apresentavam o objeto da pesquisa sem justificativa ou investigações descontextualizadas. O primeiro item, "definição clara do objeto e do objetivo em estudo" foi considerado ausente em 14 artigos (10,4\%). Somente um artigo não foi pontuado no item "quadro teórico referencial consistente e pressupostos".

Adequação da metodologia qualitativa - Encontramos três estudos (2,2\%) que não descrevem como foi feita a escolha do método qualitativo. Também poucos autores justificam com propriedade a opção por esse tipo de abordagem. Em 18 artigos (13,3\%) não havia sequer informações sobre o instrumento de pesquisa utilizado. Mesmo em muitos dos que o informaram, os itens que deveriam compor os roteiros não estavam presentes. Verificamos muita variedade na utilização dos conceitos que são específicos da pesquisa qualitativa, demonstrando certa superficialidade na utilização dos termos. Num mesmo artigo, há autores que falam de entrevista e igualmente de questionário semiestruturado para se referir ao mesmo instrumento. É importante assinalar que 11 artigos $(8,1 \%)$ sobre estudos empíricos não relatam os critérios de inclusão e de exclusão das pessoas ou grupos pesquisados.

Transparência dos procedimentos - A descrição do percurso metodológico foi tratada equivocadamente por alguns autores como um tratado teórico sobre a opção metodológica utilizada. Por exemplo, longas definições sobre representação social e poucas linhas dedicadas ao relato do caminho trilhado de fato para compreensão do objeto. Nesses casos, o problema se repetiu nas referências bibliográficas, que eram, em maioria, sobre a metodologia escolhida e não sobre o tema de pesquisa. Outra inconsistência observada nessa diretriz foi quanto à coleta de dados. Raríssimos trabalhos informam quem coletou os dados, o que impede que se avalie a relação pesquisador/pesquisado. Quanto ao critério amostral, todos que o descrevem utilizam o termo "saturação de conteúdos" para falar do número de entrevistados, sem entrar em considerações nem teóricas nem práticas sobre o significado da expressão.

Uma boa descrição do cenário do estudo e da estratégia de entrada no campo dá mais compreensão e credibilidade aos resultados. Apenas 11 (8,1\%) das 
pesquisas avaliadas em nosso estudo não pontuaram neste item. Por outro lado, $33(24,4 \%)$ não descreveram como foi feita a interlocução no campo e $46(34,1 \%)$ omitiram como as informações foram registradas. No caminho contrário, há um artigo em que o autor descreve mudanças nos instrumentos de pesquisa depois que a coleta de dados havia começado, a partir da verificação de que as estratégias adotadas não se orientavam para os objetivos, o que demonstra uma busca de transparência e de qualidade (ESPÍNDOLA; BLAY, 2009).

Os aspectos éticos estiveram ausentes em 29 (21,5\%) artigos que incluíam pesquisas com seres humanos. Em muitos, parte da descrição da metodologia se encontrava na apresentação dos resultados, por exemplo, dados sobre o roteiro de entrevista, critério de saturação, estratégia de análise dos dados. Outros informam a utilização de determinada técnica de pesquisa, sem que seu uso correto se evidencie nos resultados. Nos artigos em que foi utilizada a técnica de análise documental, frequentemente não é descrito claramente no que se baseou a análise. Assim, muitos se apresentam apenas como uma síntese comentada do que foi lido.

Solidez da abordagem interpretativa - Em 15 artigos (11,1\%), não foi descrita a forma de análise, ou seja, como foi ordenado, classificado e analisado o material. Em 105 do total de artigos (77,8\%), não foi feita a contextualização históricoespacial-social das informações recolhidas em campo, e, em 117 artigos, (86,7\%) não foram consideradas as limitações do estudo frente a vários aspectos, dentre eles, o avanço do conhecimento. Em 32 estudos (23,7\%), as interpretações não foram claras, nem apoiadas em evidências e em diálogo com a literatura pertinente ao tema. $\mathrm{O}$ texto não apresentava uma leitura fluente e uma boa comunicação em 11 artigos (8,1\%). Encontramos trabalhos que não descrevem a forma de análise textual e não constroem categorias que deveriam ser analisadas. Alguns autores simplesmente vão apresentando os resultados e os interpretando. Um fato a se destacar é que encontramos várias formas de descrição dos resultados, o que não obrigatoriamente é uma fragilidade: certos pesquisadores criam categorias bem próximas à realidade empírica; outros utilizam categorias gerais e abstratas na interpretação; outros ainda apresentam de imediato a discussão dos achados sem mostrar que passaram pelo processo classificatório.

Quanto aos campos teóricos de análise, encontramos estudos que informam a realização da abordagem hermenêutica, mas, na verdade, apresentam apenas uma análise temática. Embora teoricamente sejam distintos os conceitos, análise 
temática e de conteúdo aparecem como sinônimos na maioria dos estudos que informaram essas abordagens. O termo triangulação é utilizado em diferentes situações: quando se usam várias técnicas (entrevista + grupo focal + observação); quando se usam vários métodos (qualitativos, quantitativos, estudos clínicos); quando profissionais de diferentes áreas analisam o mesmo objeto. Essa expressão, "triangulação", é também utilizada na literatura de forma polissêmica, conforme Minayo (2013) e Denzin (1970).

Por fim, em alguns artigos, verificamos que os autores dão destaque nos resultados de seus trabalhos a certos depoimentos de interlocutores que não têm relação ou até divergem dos pressupostos iniciais, o que não constitui um problema. Entretanto, essas mudanças de perspectiva não são comentadas, problematizadas ou valorizadas na síntese interpretativa do estudo.

\section{Discussão}

Dentro dos padrões de cientificidade, um artigo qualitativo deve, de um lado, ser avaliado dentro das mesmas exigências metodológicas que convêm a qualquer trabalho acadêmico: que tenha objeto e objetivos claros, que se apoie em revisão da bibliografia e em teorias reconhecidas, que apresente procedimentos metodológicos condizentes, que explicite os critérios classificatórios, que mostre resultados baseados nos dados empíricos, interagindo com as reflexōes científicas já existentes sobre o assunto de forma contextualizada, e, por fim, que explicite claramente também as limitaçôes do estudo. De outro lado, é importante que cada etapa do ciclo de conhecimento se atenha às especificidades da pesquisa qualitativa, que frequentemente se diferenciam dos procedimentos dos estudos quantitativos. Portanto, é preciso conhecer as lógicas de ambas e buscar o rigor científico que cada uma deve ter.

Um dos pontos mais críticos a respeito da cientificidade do método é a não pretensão de generalização de seus dados, por causa de sua peculiaridade e proposta de conhecimento. Embora isso seja verdade, Collingridge e Gantt (2008), da Intermountain Healthcare, Salt Lake City, Utah, EUA, destacam que é possível generalizar resultados de estudos em contextos semelhantes com certo grau de confiança, e comparam com a jurisprudência na área de Direito, onde, num precedente aplicável, a generalização é estabelecida através da análise das características semelhantes entre casos particulares. 
Outra crítica comum de quem trabalha com método quantitativo diz respeito à confiabilidade dos estudos, ou seja, a não pretensão de sua reprodutibilidade. Sobre isso, Collingridge e Gantt (2008) ressaltam que a pesquisa qualitativa não minimiza a importância de encontrar resultados semelhantes em contextos semelhantes, e, em geral, isso ocorre. Mas, quando se encontram divergências, não significa que o método qualitativo não seja confiável, pois ele não busca a verdade, que, muitas vezes, de forma sofismável, é tratada como quantificação da realidade. $\mathrm{O}$ que a pesquisa qualitativa procura insistentemente é compreender e interpretar da forma mais fiel possível a lógica interna dos sujeitos que estuda e dar conhecimento de sua "verdade" (MINAYO, 2012b). Diferenças de interpretação frequentemente refletem uma compreensão multifacetada de fenômenos sociais complexos. Alguns autores medem a eficácia de suas pesquisas através das surpresas que experimentam quando vão a campo (OLIVEIRA, 2012), sempre partindo de estudos anteriores, quando existem.

Nesta análise, encontramos um percentual maior de artigos considerados consistentes, seguindo as diretrizes RATS, do que outros autores que também elaboraram revisões. Numa análise sistemática de estudos qualitativos sobre a percepção de familiares sobre anorexia e bulimia, dos 58 artigos, Espíndola e Blay (2009), do Departamento de Psiquiatria da Escola Paulista de Medicina, elencaram apenas nove (menos de 20\%) seguindo critérios que se assemelham às diretrizes RATS quanto aos aspectos metodológicos.

Quanto aos estudos pouco consistentes, interpretaçôes simplistas em investigações mal elaboradas já haviam sido destacadas por vários cientistas sociais, como Minayo (2012a), em levantamento sobre trabalhos qualitativos de autores brasileiros, Gomes e Silveira (2012) em ensaio crítico, e Canesqui (2011), em artigo de revisão. Os diversos problemas já citados poderiam ser minimizados com melhor formação dos autores, que incluísse reflexões sobre os fundamentos teóricos e filosóficos que embasam o método e sobre como contextualizar os estudos. $\mathrm{O}$ desenvolvimento de pesquisas qualitativas depende muito da competência do investigador. Por isso, a polissemia que vem do uso superficial dos conceitos e a redução do método às técnicas são consideradas problemas cruciais da pesquisa qualitativa (BOSI, 2012). Quem não conhece bem os conceitos com os quais está trabalhando terá dificuldade tanto para criar os instrumentos adequados como para realizar um trabalho intersubjetivo em 
campo ou para fazer uma boa análise compreensiva e interpretativa $(\mathrm{COHN}$, 2013; MINAYO, 2013; ONOCKO-CAMPOS, 2011). Bradley, Curry e Devers (2007), do Departamento de Epidemiologia e Saúde Pública da Faculdade de Medicina da Universidade de Yale, EUA, conferem um papel importante nesse sentido aos periódicos científicos, concordando com o que concluímos neste estudo, sobre a necessidade de instruções claras e abertura para atender às especificidades dessa abordagem.

Corroborando as limitações que encontramos no material estudado, Weiner et al. (2011), da Escola de Saúde Pública Global da Universidade da Carolina do Norte em Chapel Hill, EUA, em revisão de pesquisas qualitativas sobre serviços de saúde, relatam que metade apresentava pouco ou nenhum detalhe sobre aspectoschave do método e somente $17 \%$ dos artigos descreviam como foram feitas a coleta e a análise dos dados. Essa falta de clareza contribui para o menosprezo de estudos dessa natureza. Por exemplo, do ponto de vista da retidão dos procedimentos, raríssimos estudos abordam questões concernentes à relação entre o pesquisador e seu interlocutor, deixando dúvidas quanto ao caráter ético do estudo e ignorando as implicações dessa relação, principalmente quando os pesquisadores estudam seus próprios pacientes (BRADLEY; CURRY; DEVERS, 2007).

A solidez compreensiva e interpretativa responsável por desvendar a "lógica interna dos sujeitos" também é um ponto fraco de muitos artigos qualitativos. No entanto, resultados piores foram encontrados por Yamazaki et al. (2009), professores da Faculdade de Humanidades e Sociologia da Universidade de Tóquio, no Japão. Em estudo de revisão crítica, não encontraram informação sobre o tipo de análise realizada em pelo menos 41\% dos artigos. Sofaer (2002), pesquisador da Escola de Assuntos Públicos em Nova Iorque, EUA, refere que a análise dos dados é um grande desafio, pois as informações de campo são tipicamente sugestivas e raramente conclusivas. Entretanto, o processo de análise, ressalta o autor, precisa ser altamente refletido, discutido e sistematizado. Ainda nessa mesma linha de pensamento sobre as fragilidades dos estudos qualitativos, Sandelowski e Barroso (2003), da Universidade da Carolina do Norte, EUA, propõem que os resultados de estudos qualitativos na área da saúde possam ser classificados em um continuum que reflita sua qualidade: "nenhuma descoberta", "pesquisa tópica”, "survey temático", "descrição conceitual ou temática” ou "explicação interpretativa”, e assim conseguir diferenciar artigos consistentes dos inconsistentes. 
Ponto importante a ressaltar é a evidência de que vem ocorrendo maior abertura para aceitação de publicações de cunho qualitativo em revistas da área médica. Isso acontece por vários motivos. Entre eles, dois são fundamentais: o reconhecimento da relevância das questôes sociais e psicológicas no desenvolvimento das doenças e a consciência dos sujeitos sobre seus direitos associada à pressão para que sejam ouvidos na solução de seus problemas (MINAYO, 1998). Shuval et al. (2011), integrantes da Escola de Assuntos Públicos em Nova Iorque, EUA, argumentam, contudo, que o percentual de incremento de estudos e publicações ainda é muito pequeno. Nesse sentido, os editores científicos têm papel fundamental. Devers (1999), investigador da Agência de Pesquisa e Política Pública em Saúde de Rockville, EUA, destaca que os critérios tradicionais de avaliação da qualidade dos textos científicos ainda estão enraizados na perspectiva filosófica positivista, estreitamente associada a exigências estatísticas e matemáticas. $\mathrm{O}$ autor sugere que orientações adequadas para induzir e avaliar as pesquisas qualitativas reforçarão esse campo e facilitarão uma disposição maior de financiamento e publicação do conhecimento gerado por elas. Os que adotam política editorial inclusiva de artigos qualitativos publicam mais e oferecem maiores chances de aperfeiçoamento aos autores.

Nesse sentido, é muito louvável que revistas de elevado impacto como Lancet e JAMA tenham se aberto para a divulgação de estudos qualitativos, embora o número de artigos aceitos ainda seja muito pequeno e os pareceristas precisem ser escolhidos entre os que compreendem a lógica desses estudos e trabalhem efetivamente a partir dessa perspectiva (COLLINGRIDGE; GANTT, 2008).

\section{Conclusões}

Concluímos dizendo que, na área médica, a pesquisa qualitativa é importante para a ampliação do conhecimento clínico e melhora da qualidade do atendimento. Ela é adequada para a compreensão de fenômenos dentro de seu contexto, estabelecendo ligaçôes entre conceitos, representações, crenças e comportamentos, gerando ricas informações para tomada de decisão médica em intersubjetividade com os pacientes. Os estudos qualitativos também podem revelar insights críticos que ajudam a lidar com as deficiências do sistema de saúde.

Por fim, cumpre-nos destacar que este estudo tem um limite muito claro: analisa apenas a produção qualitativa de médicos publicada em periódicos nacionais indexados e se restringe às palavras-chave (citadas na parte do método) que dão acesso às obras dos autores que nelas publicam. Certamente, há artigos veiculados 
em revistas locais e não indexadas a que, neste estudo, não tivemos acesso. Apesar desses limites, ressaltamos a evidência de que já existe uma tradição no uso da metodologia qualitativa por médicos pesquisadores. Observamos que esse uso é ainda marginal e polêmico, pois são poucos os autores médicos no universo das publicações de estudos qualitativos. $\mathrm{Na}$ área da saúde a pesquisa quantitativa é hegemônica. Contudo, inferimos que é possível fortalecer a consolidação dos estudos, conhecendo em detalhes as dificuldades e acreditando nas possibilidades. ${ }^{1}$

\section{Referências}

BIOMED CENTRAL. Qualitative research review guidelines - RATS. Disponível em: <http://www.biomedcentral.com/authors/rats>. Acesso em: 04 jun. 2014.

BOSI, M. L. M. Pesquisa qualitativa em saúde coletiva: panorama e desafios. Ciência \& Saúde Coletiva, v. 17, n. 3, p. 575-586, 2012.

BRADLEY, E. H.; CURRY, L. A.; DEVERS, K. J. Qualitative data analysis for health services research: developing taxonomy, themes, and theory. Health Serv. Res., v. 42, n. 4, p. 1758-1772, 2007.

CANESQUI, A. M. Sobre a presença das ciências sociais e humanas na saúde pública. Saúde e Sociedade, v. 20, n. 1, p. 16-21, 2011.

COHN, A. Ciências Sociais e Saúde Pública/Coletiva: a produção do conhecimento na sua interface. Saúde Soc., São Paulo, v. 22, n. 1, p. 15-20, 2013.

COLLINGRIDGE, D. S.; GANTT, E. E. The quality of qualitative research. American Journal of Medical Quality, v. 23, p. 389-395, 2008.

DENZIN, N. K. The research act. Chicago: Aldine Publishing, 1970.

DEVERS, K. J. How will we know "good" qualitative research when we see it? Beginning the dialogue in health services research. Health Serv. Res, v. 34, p. 1153-1188, 1999.

ESPÍNDOLA, C. R.; BLAY, S. L. Percepção de familiares sobre a anorexia e bulimia: revisão sistemática. Rev. Saúde Pública, v. 43, n. 4, p. 707-716, 2009.

GOMES, M. H. A.; SILVEIRA, C. Sobre o uso de métodos qualitativos em Saúde Coletiva, ou a falta que faz uma teoria. Rev. Saúde Pública, v. 46, n. 1, p. 160-165, 2012.

GUERRIERO, I. C. Z.; MINAYO, M. C. S. O desafio de revisar aspectos éticos das pesquisas em ciências sociais e humanas: a necessidade de diretrizes específicas. Physis: Revista de Saúde Coletiva. Rio de Janeiro, v. 23, n. 3, p. 763-782, 2013.

HOFF, T. J.; WITT, L. C. Exploring the use of qualitative methods in published health services and management research. Medical Care Research and Review, v. 57, p. 139-160, 2000. MARSIGLIA, E. M. G. Temas emergentes em ciências sociais e saúde pública/coletiva: a produção do conhecimento na sua interface. Saúde Soc. São Paulo, v. 22, n. 1, p. 32-43, 2013. 
MCKIBBON, K.; GADD, C. S. A quantitative analysis of qualitative studies in clinical journals for the 2000 year. BMC Medical Informatics and Decision Making, v. 4, n. 11, 2004. MINAYO, M. C. S. O desafio do conhecimento: Pesquisa qualitativa em saúde. São Paulo: Hucitec, 2013.

. Herança e promessas do ensino das Ciências Sociais na área da Saúde. Cad. Saúde Pública, v. 28, n. 12, p. 2367-2372, 2012a.

MINAYO, M. C. S. Análise qualitativa: teoria, passos e fidedignidade. Ciênc. saúde coletiva, v. 17 , n. 3, p. 621-626, 2012b.

. Construção da identidade da antropologia na área da saúde: o caso brasileiro. In: . Antropologia e saúde: traçando identidades e explorando fronteiras. Rio de Janeiro: Relume Dumará, 1998. p. 29-43.

MORSE, J. M. Reconceptualizing Qualitative Evidence. Qual. Health Res., v. 16, n. 3, p. 415-422, 2006.

OLIVEIRA, A. L. O. et al. Sobre fazer ciência na pesquisa qualitativa: um exercício avaliativo. Rev. Saúde Pública, v. 46, n. 2, p. 392-394, 2012.

ONOCKO-CAMPOS, R. T. Fale com eles! O trabalho interpretativo e a produção de consenso na pesquisa qualitativa em saúde: inovações a partir de desenhos participativos. Physis: Revista de Saúde Coletiva. Rio de Janeiro, v. 21, n. 4, p. 1269-1286, 2011.

SANDELOWSKI, M.; BARROSO, J. Classifying the findings of qualitative studies. Qual. Health Res, v. 13, p. 905e23, 2003.

SHUVAL, K. et al. Is Qualitative Research Second Class Science? A quantitative longitudinal examination of qualitative research in medical journals. PLoS One, v. 6, n. 2, p. e16937, 2011. SOFAER, S. Qualitative research methods. International Journal for Quality in Health Care, v. 14, p. 329-336, 2002.

TAQUETTE, S. R.; MINAYO, M. C. S.; RODRIGUES, A. O. The perceptions of medical researchers on qualitative methodologies. Cad. Saúde Pública, v. 31, n. 4, p. 1-11, 2015.

TURATO, E. R. Métodos qualitativos e quantitativos na área da saúde: definições, diferenças, e seus objetos de pesquisa. Rev. Saúde Pública. São Paulo, v. 39, n. 3, p. 507-14, 2005.

WEINER, B. J. et al. Use of qualitative methods in published health services and management research: a 10-year review. Med Care Res Rev, v. 68, n. 1, p. 3-33, 2011.

YAMAZAKI, $\mathrm{H}$. et al. Characteristics of qualitative studies in influential journals of general medicine: a critical review. BioScience Trends, v. 3, n. 6, p. 202-209, 2009.

\section{Nota}

${ }^{1}$ S. R Taquette e M. C. S Minayo participaram igualmente da coleta e interpretação dos dados, redação e aprovação da versão final do artigo. 
Analysis of qualitative studies conducted by physicians and published in Brazilian scientific journals between 2004 and 2013

We analyze articles resulting from research by physicians using qualitative methods. The bibliographic search was conducted in the SciELO Brazil from 2004 to 2013 with the keywords: qualitative study, interview, focus group, participant observation, content and speech analysis, social representation, hermeneutic-dialectic. We approach the texts through a theoretical construct based on RATS guidelines for review qualitative studies. We classified the material as: consistent, somewhat consistent and inconsistent. A total of 135 articles in 28 periodicals were selected. We evaluated most articles as consistent (64.4\%). The main problems found were: lack of information of the methodological approach; partial descriptive analysis of data without dialogue with literature; conclusions that go no further than common sense; results lacking context and no consideration of limitations on the study. We concluded that the most articles are scientifically valid and we suggest that journals of the health area include specific guidelines in their editorial standards for this type of publication.

> Key words: qualitative study; scientific knowledge; epistemology; research peer review. 